Supplement of

\title{
SoilKsatDB: global database of soil saturated hydraulic conductivity mea- surements for geoscience applications
}

\section{Surya Gupta et al.}

Correspondence to: Surya Gupta (surya.gupta@usys.ethz.ch)

The copyright of individual parts of the supplement might differ from the article licence. 


\section{Ksat data tutorial}

Surya Gupta, Tom Hengl, Peter Lehmann, Sara Bonetti, Dani Or

- Installing and loading packages

- Temperate climate region Ksat values PTF

- Temperate Ksat PTF tested on tropical dataset

- Ksat PTF developed for Lab measurements soil samples using RF

- Lab PTF tested on field dataset

We prepared a comprehensive global compilation of measured Ksat training point data $(N=13,258)$ called "SoilKsatDB" by importing, quality controlling, and standardizing tabular data from existing soil profile databases and legacy reports. The SoilKsatDB was used to develop the pedotransfer functions (PTFs) for temperate climate region and lab-based measured soil samples. These PTFs were applied to tropical climate region and field-based measurements, respectively to evaluate the suitability for other regions. Here, the objective of this report to show the methods used to develop the PTFs with R code and stepwise description. This document also shows that how we connect the Ksat dataset with metadata and climate zone dataset.

SoilKsatDB link

To cite this dataset please use:

Gupta, S., Hengl, T., Lehmann, P., Bonetti, S., and Or, D.: SoilKsatDB: global soil saturated hydraulic conductivity measurements for geoscience applications, Earth Syst. Sci. Data Discuss.,, in review, 2021.

Examples of using the SoilKsatDB to generate global maps of Ksat can be found in:

Gupta, S., Lehmann, P., Bonetti, S., Papritz, A. and Or, D.: Global prediction of soil saturated hydraulic conductivity using random forest in a Covariate-based Geo Transfer Functions (CoGTF) framework. manuscript accepted to Journal of 
\#\# Loading required package: viridisLite

\section{library (ranger)}

library (mlr)

\#\# Loading required package: ParamHelpers

\#\#

\#\# Attaching package: 'ParamHelpers'

\#\# The following object is masked from 'package:raster':

\#\#

\#\# getValues

\#\# 'mlr' is in maintenance mode since July 2019. Future development

\#\# efforts will go into its successor 'mlr3' (<https://mlr3.mlr-org.com〉).

\#\#

\#\# Attaching package: 'mlr'

\#\# The following object is masked from 'package:raster':

\#\#

\#\# resample

library (randomForest)

\#\# randomForest $4.6-14$

\#\# Type rfNews() to see new features/changes/bug fixes.

\#\#

\#\# Attaching package: 'randomForest' 
\#\# The following object is masked from 'package:ranger':

\#\#

\#\# importance

\section{Temperate climate region Ksat values PTF}

Here, we loaded the Ksat dataset and extracted the temperate climate region based Ksat values. The soil samples were converted into log scale and then selected points with available three soil basic properties (sand, clay and bulk density).

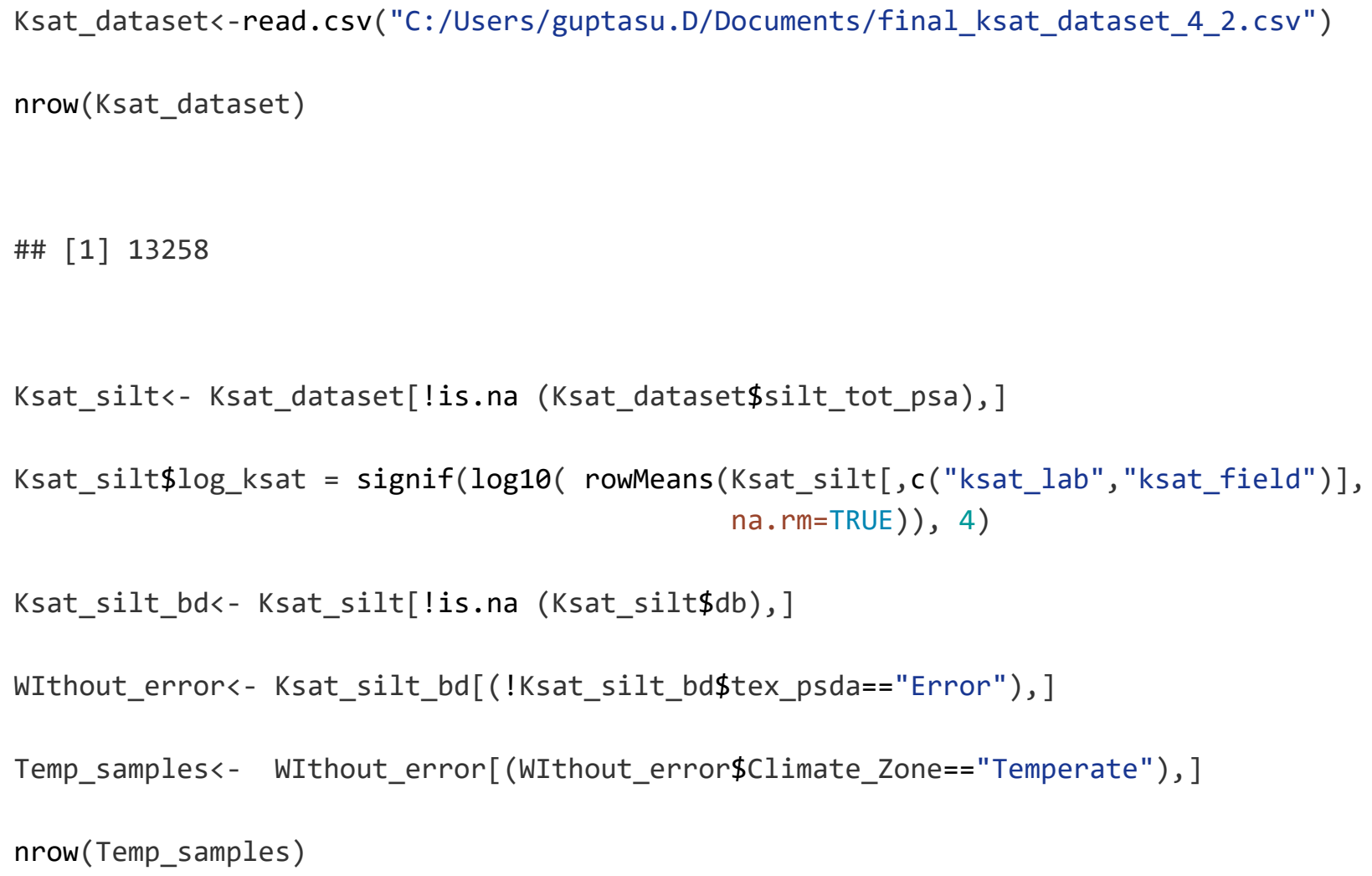




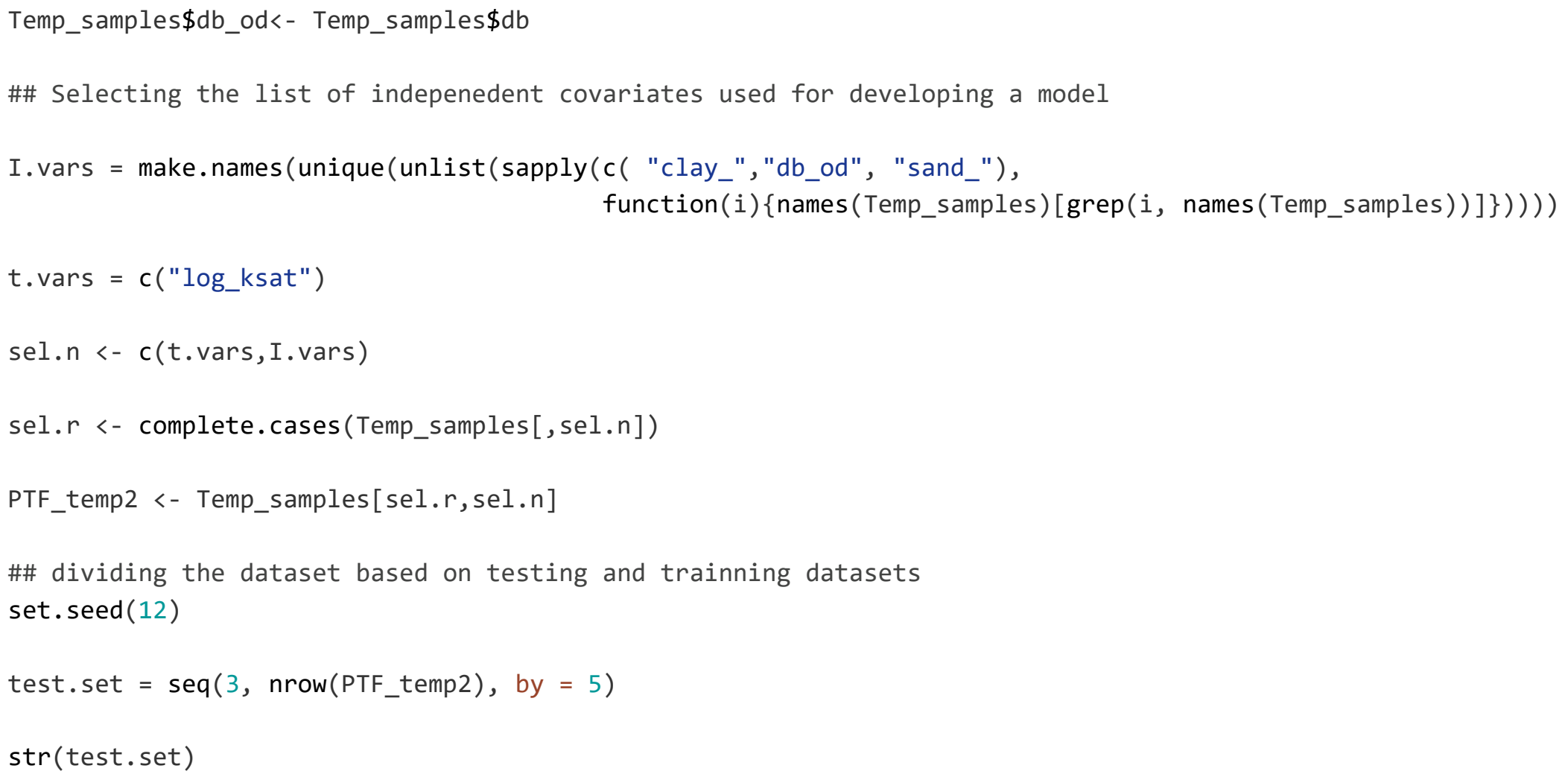


learner = mlr::makeLearner("regr.ranger", mtry =3, num.trees=85)

tsk <- mlr::makeRegrTask (data = PTF_temp2, target $=$ t.vars, blocking $=$ NULL)

m <- mlr::train(learner, tsk, subset = training.set)

\#\#Predction for the testing dataset

$\mathrm{p}=\operatorname{predict}(\mathrm{m}$, newdata $=\mathrm{PTF}$ temp2[test.set, $])$

comparison <- p\$data

\#\#Linear model between predictions and measurements

gg<- $\operatorname{lm}($ comparison\$truth comparison\$response)

$\operatorname{summary}(g g)$

\#\#

\#\# Call:

\#\# $\operatorname{lm}($ formula = comparison $\$$ truth $\sim$ comparison $\$ r e s p o n s e)$

\#\#

\#\# Residuals:

\#\# Min $1 Q$ Median $3 Q \quad$ Max

$\begin{array}{llllll}\# \# & -3.10753 & -0.26038 & 0.01586 & 0.32553 & 2.99783\end{array}$

\#\#

\#\# Coefficients:

\#\# Estimate Std. Error $t$ value $\operatorname{Pr}(>|t|)$

\#\# (Intercept) $\quad 0.10886 \quad 0.04381 \quad 2.485 \quad 0.0131$ *

\#\# comparison\$response $0.95042 \quad 0.0196948 .264<2 \mathrm{e}-16 * * *$

\#\# - - -

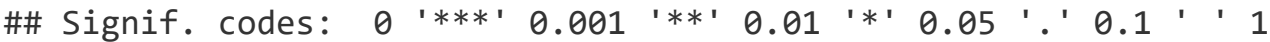

\#\#

\#\# Residual standard error: 0.6431 on 1657 degrees of freedom

\#\# Multiple R-squared: 0.5843, Adjusted R-squared: 0.5841

\#\# F-statistic: 2329 on 1 and 1657 DF, p-value: < 2.2e-16 
\#\#CCC

ccc = DescTools: :CCC (comparison\$truth, comparison\$response, $\mathrm{ci}=$ "z-transform", conf.level $=0.95$, na.rm=TRUE) $\$ r h o . c$

CCC
\#\#
est lwr.ci
upr.ci

$\begin{array}{llllll}\text { \#\# } & 1 & 0.7466269 & 0.7257605 & 0.7661209\end{array}$

\#\#bias

bias (comparison\$response, comparison\$truth)

\#\# [1] -0.005981836

\#\#RMSE

rmse(comparison\$truth, comparison\$response)

\#\# [1] 0.6439256

\#\#Transform log10 to normal values

comparison $\$ \log 10 \mathrm{ksat} 1<-10^{\wedge}$ comparison $\$$ truth

comparison\$response $1<-10^{\wedge}$ comparison $\$$ response 
\#\#Hexbin Plot

hexbinplot(log10ksat1 response1,

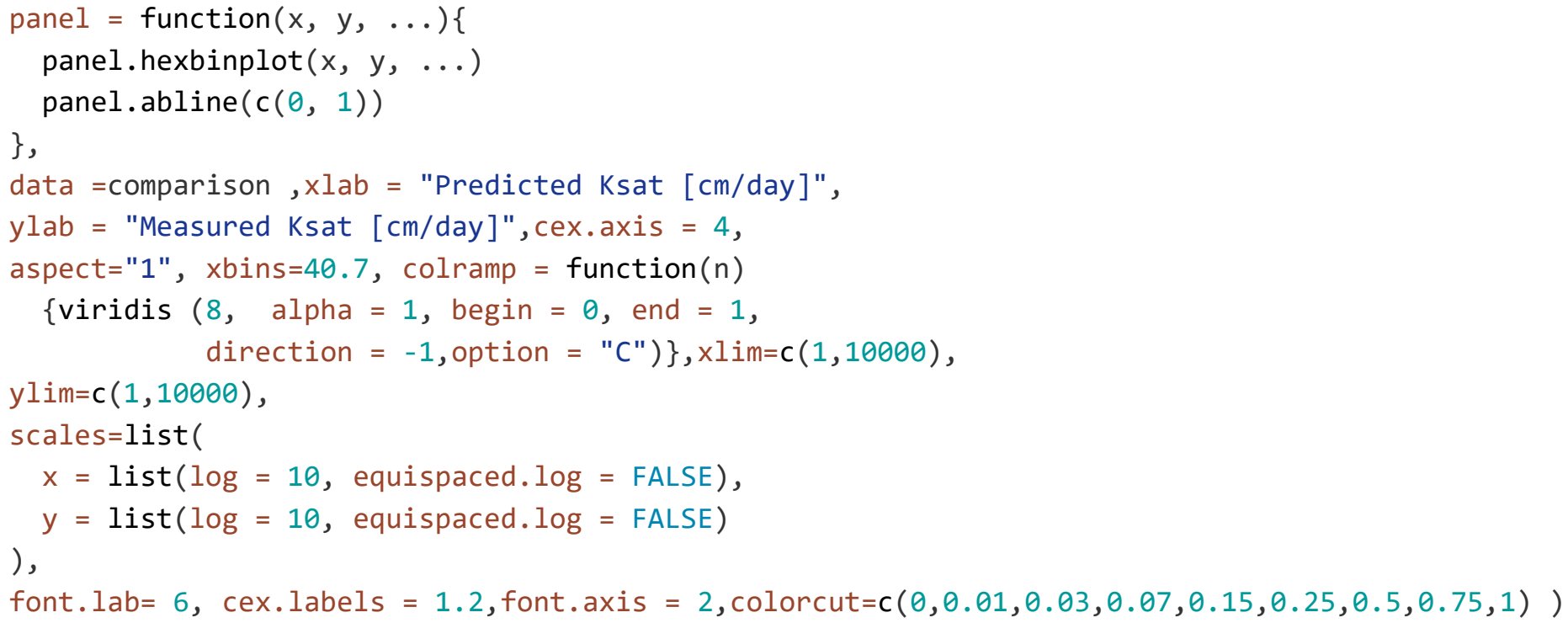




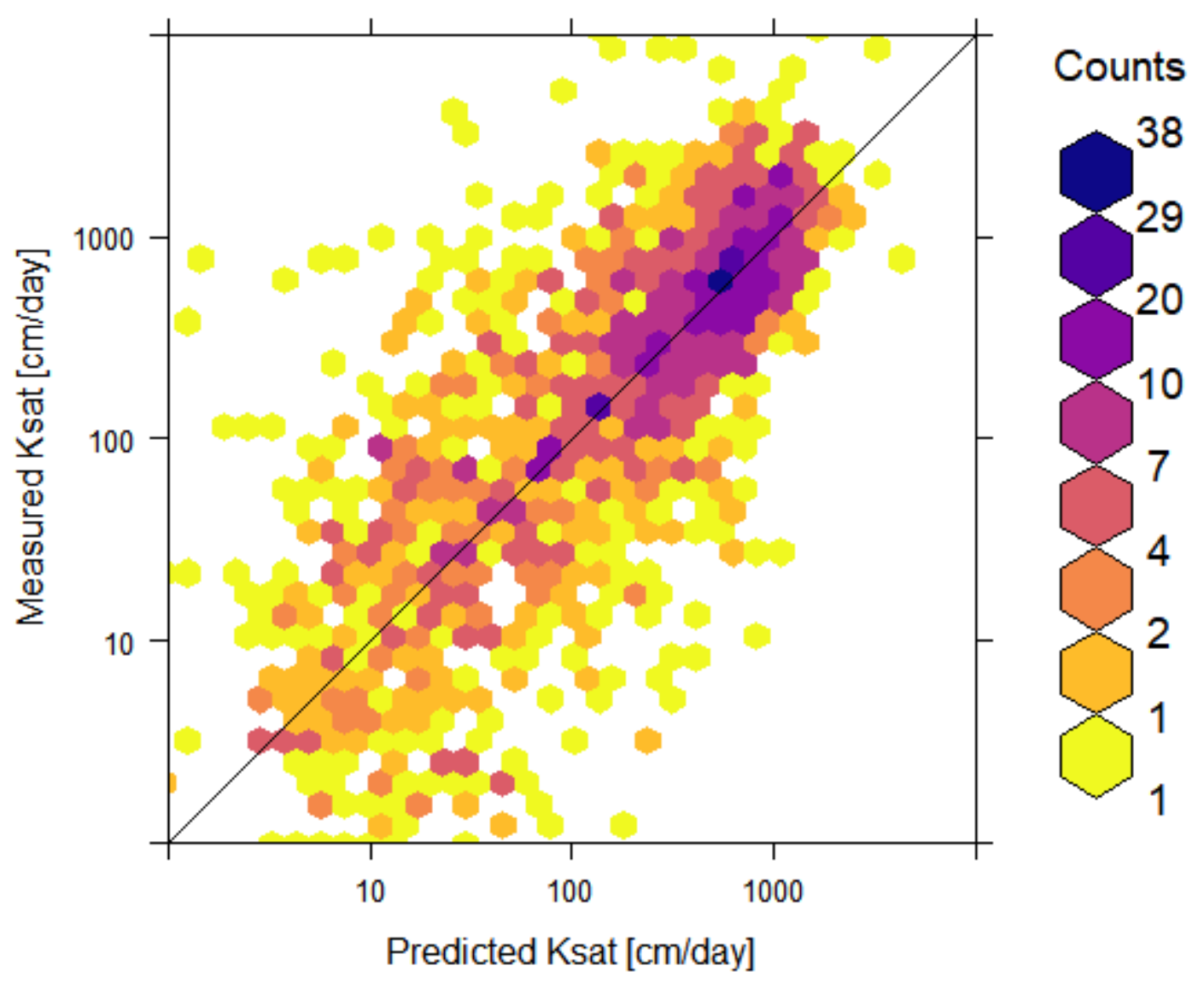

\#\#variable importance_selection

Var.ksat <- as.formula (paste("log_ksat clay_tot_psa + db_od + sand_tot_psa")) Var.ksat

\#\# log_ksat clay_tot_psa + db_od + sand_tot_psa 


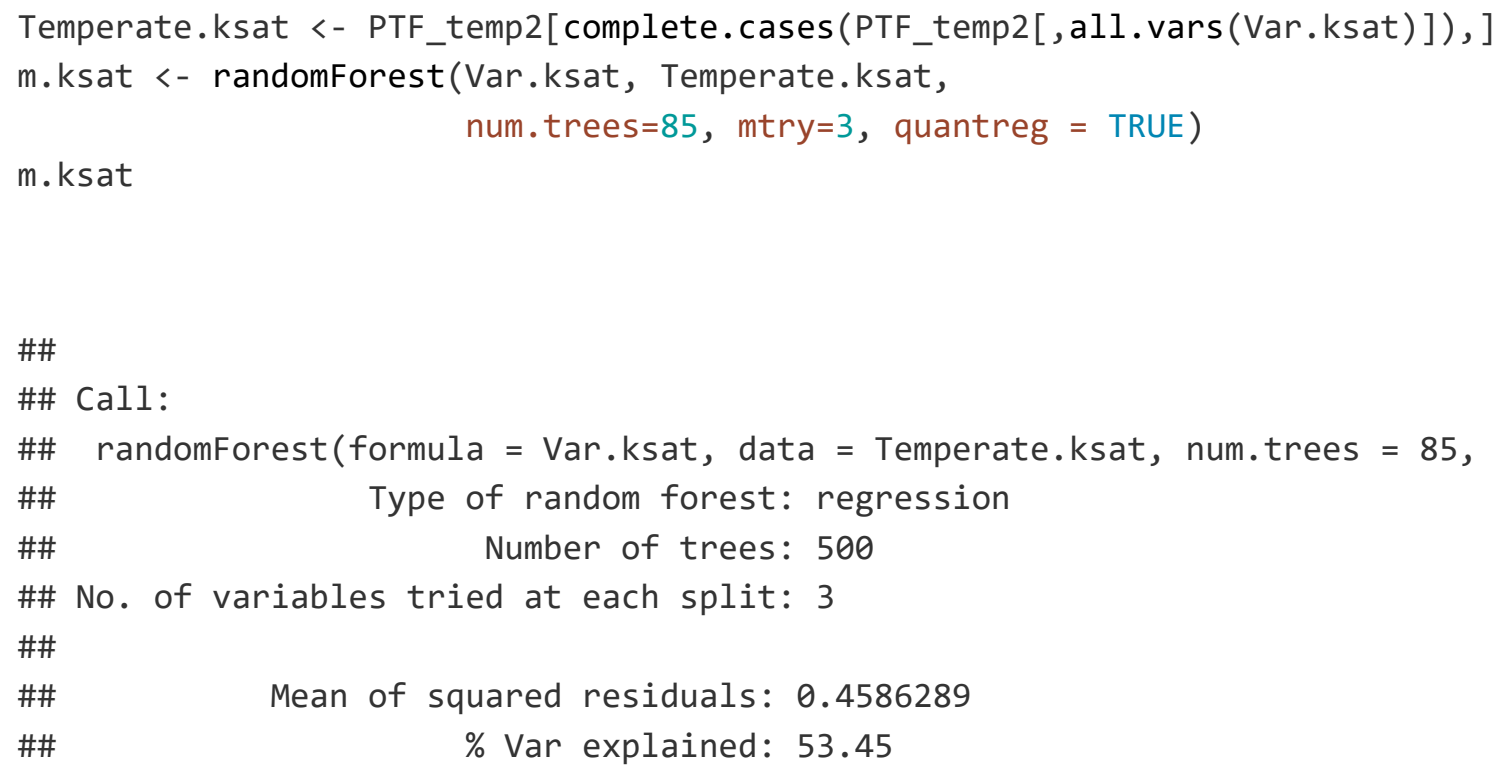




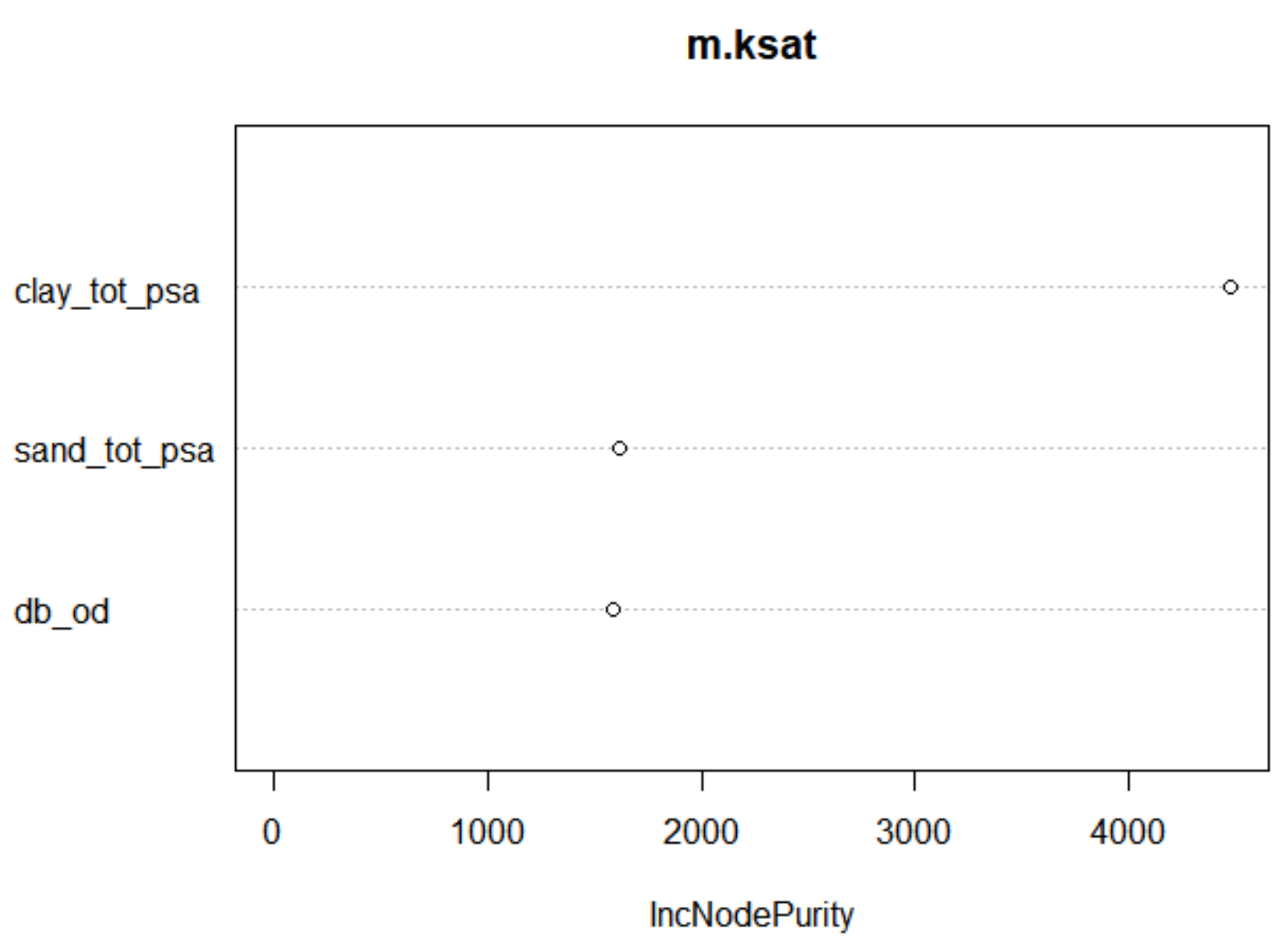

\section{Temperate Ksat PTF tested on tropical dataset}

PTF developed for the temperate region using RF used to predict the ksat values for tropical soil samples.

Trop_samples<- WIthout_error[(WIthout_error\$Climate_Zone=="Tropical"), ]

nrow(Trop_samples) 
set. seed(12)

Trop_samples $\$ d b \_o d<-$ Trop_samples $\$ d b$

$p=\operatorname{predict}(m$, newdata $=$ Trop_samples $)$

comparison $<-p \$ d a t a$

$y<-$ cbind(comparison, Trop_samples)

\#\#CCC

ccc $=$ DescTools: :CCC $(y$ log_ksat, $y \$ r e s p o n s e$,

$\mathrm{ci}=$ "z-transform", conf.level $=0.95$, na.rm=TRUE)\$rho.c

$\mathrm{CCC}$

\#\# est lwr.ci upr.ci

\#\# $\begin{array}{llll}1 & 0.4873967 & 0.4423483 & 0.5299919\end{array}$

\#\#bias

bias (y $\$$ response, $y \$ \log$ ksat)

\#\# [1] -0.1995795

\#\#RMSE 
rmse(y\$log_ksat, y\$response)

\#\# [1] 0.9414729

\#\#Transform log10 to normal values

y\$log_ksat1<- 10^y\$log_ksat

y\$response1<- $10^{\wedge} \mathrm{y} \$$ response

hexbinplot(log_ksat1 response1,

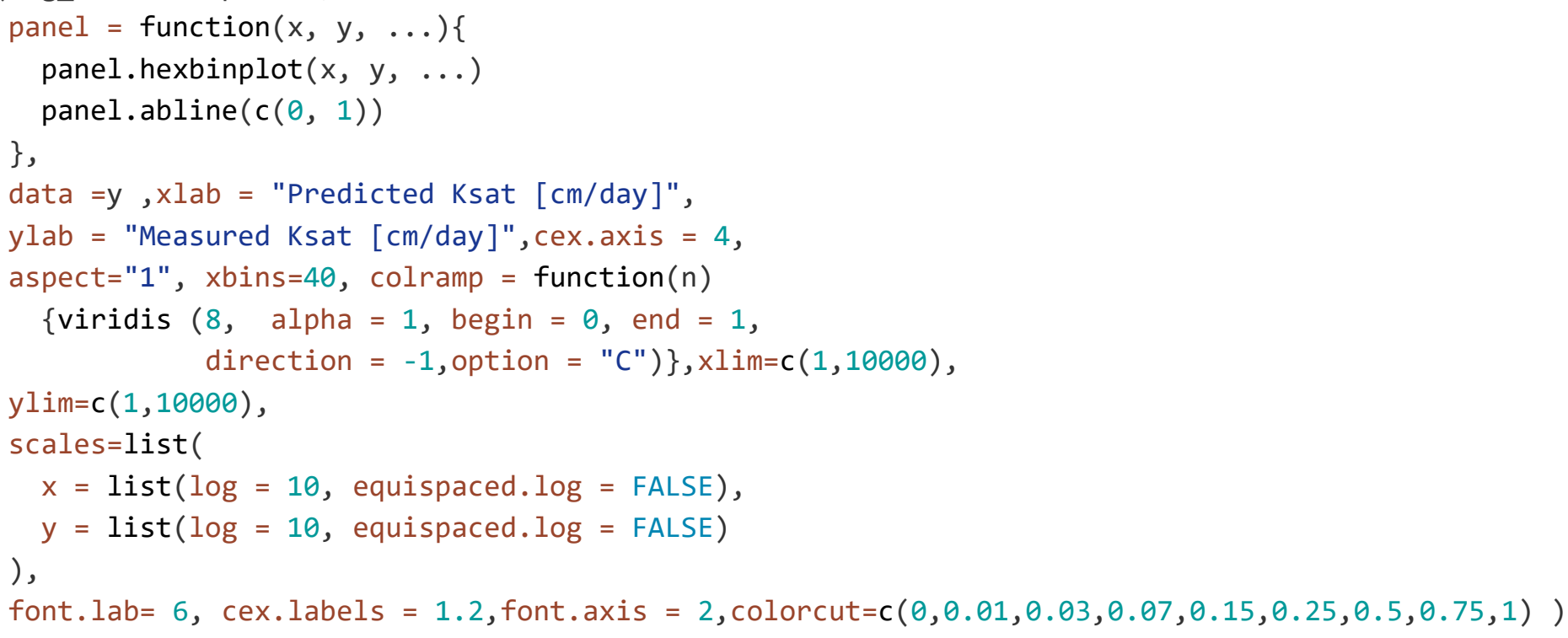




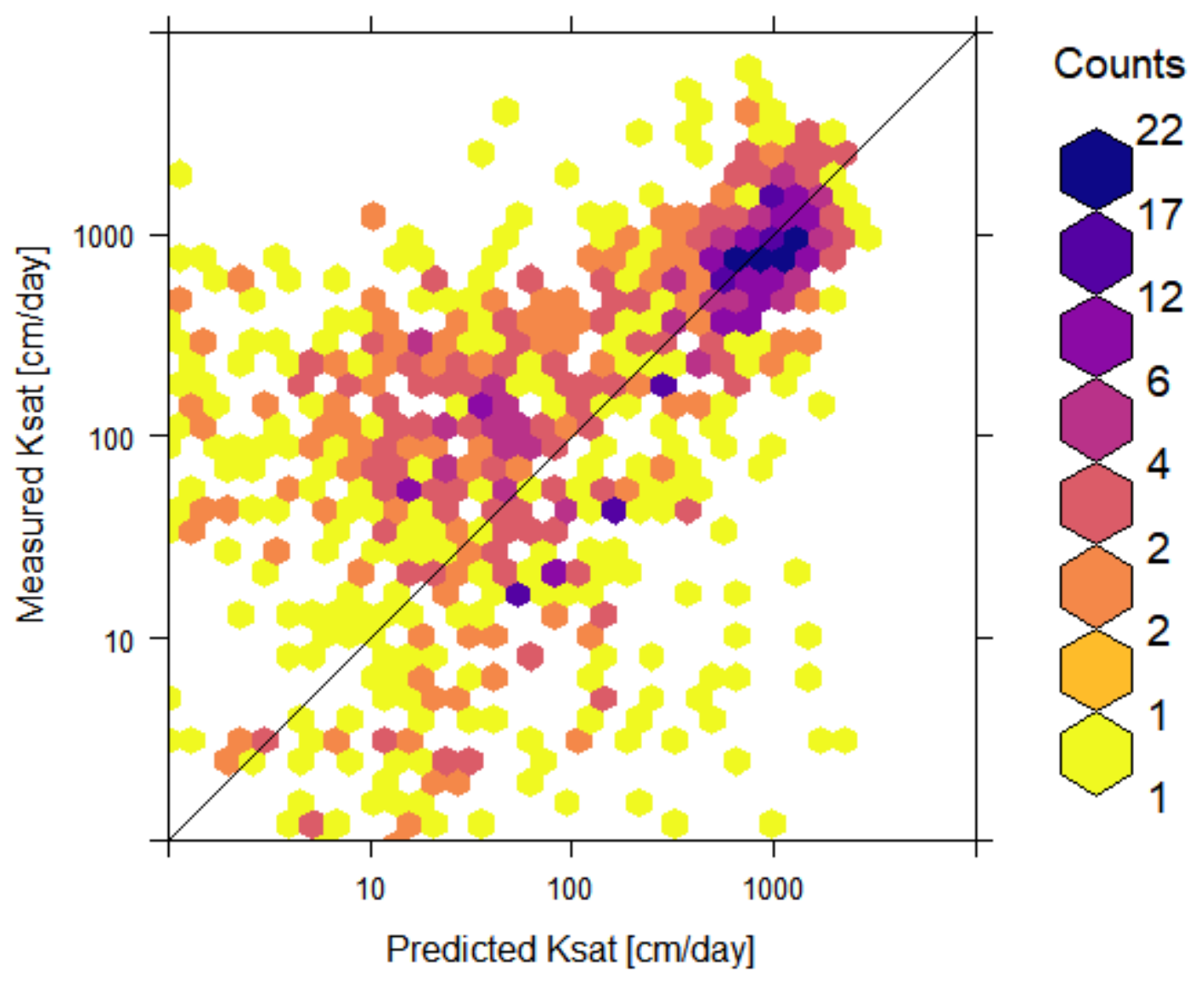

\title{
Ksat PTF developed for Lab measurements soil samples using RF
}

\author{
Ksat_dataset<-read.csv("C:/Users/guptasu.D/Documents/final_ksat_dataset_4_2.csv") \\ Ksat_silt<-Ksat_dataset[! is.na (Ksat_dataset\$silt_tot_psa), ] \\ Ksat_lab<- Ksat_silt[! is.na (Ksat_silt\$ksat_lab), ] \\ Ksat_lab\$log_ksat = signif(log10( rowMeans $($ Ksat_lab[, c("ksat_lab", "ksat_field")],
}


Ksat_lab_bd<-Ksat_lab[! is.na (Ksat_lab\$db), ]

WIthout_error<- Ksat_lab_bd[(!Ksat_lab_bd\$tex_psda=="Error"), ]

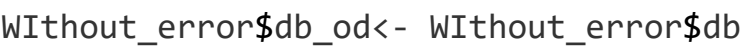

I.vars = make.names(unique(unlist (sapply(c( "clay_","db_od", "sand_"),

function(i) $\{$ names(WIthout_error) $[\operatorname{grep}(i$, names(WIthout_error)) $]\})))$ )

t.vars $=c($ "log_ksat")

sel.n $<-c($ t.vars, I.vars $)$

sel.r <- complete.cases(WIthout_error[, sel.n])

PTF_lab <- WIthout_error[sel.r, sel.n]

nrow(PTF_lab)

\#\# [1] 8491

\#\# dividing the dataset based on testing and trainning datasets set. $\operatorname{seed}(12)$

test.set $=\operatorname{seq}(3, \operatorname{nrow}($ PTF_lab), by $=5)$

str(test.set)

\#\# num [1:1698] $3 \begin{array}{lllllllllll}8 & 13 & 18 & 23 & 28 & 33 & 38 & 43 & 48 & \ldots\end{array}$ 


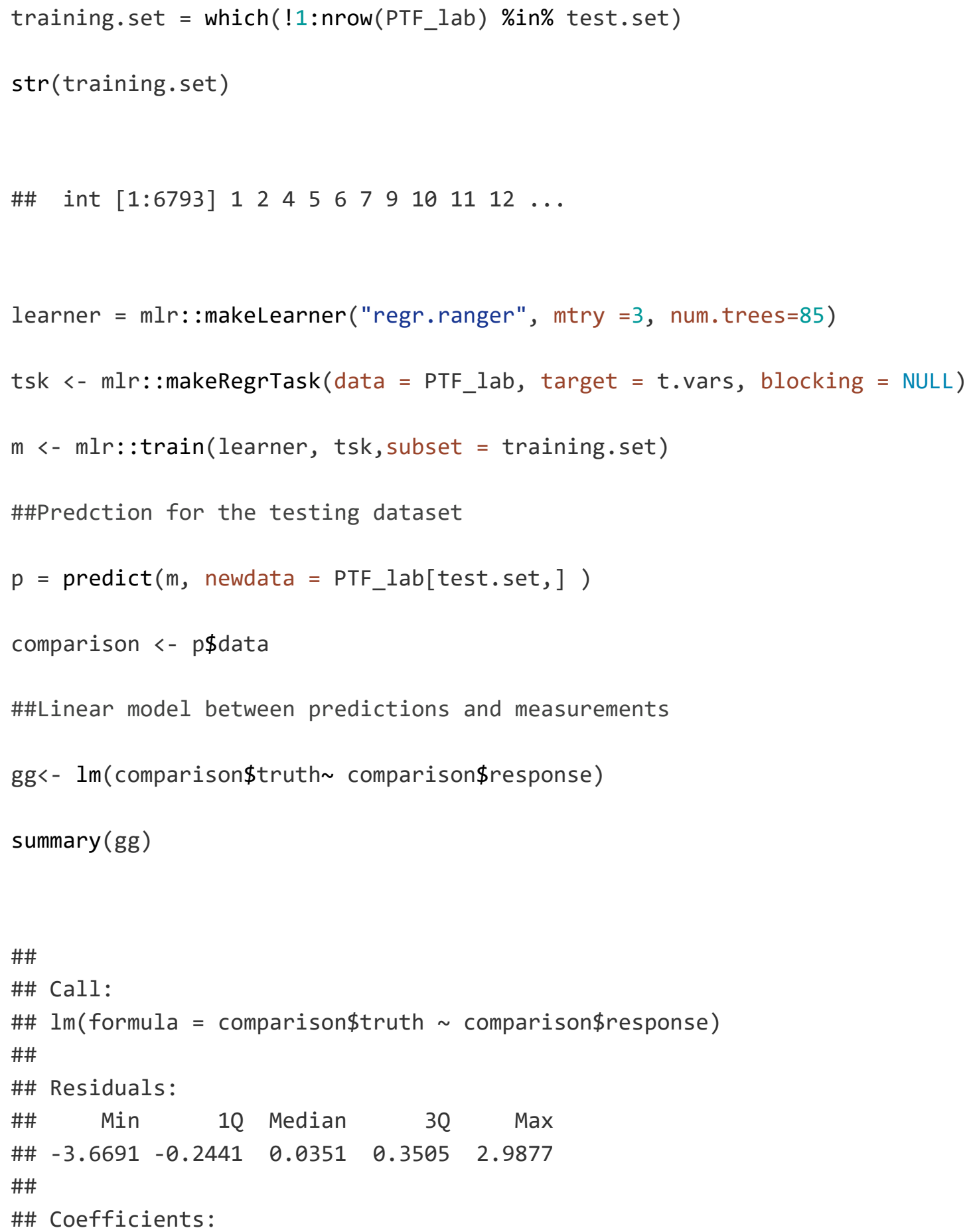




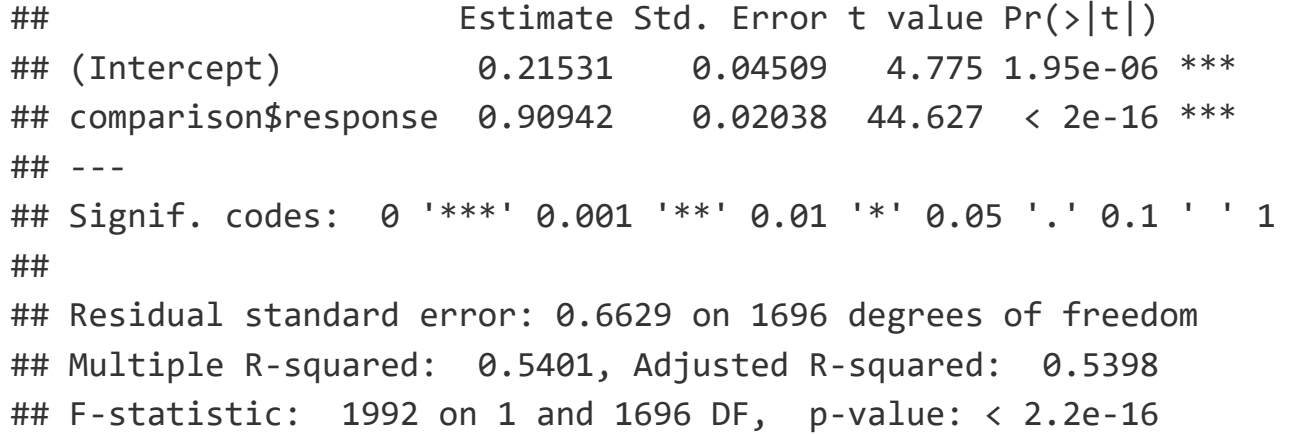

\#\#CCC

ccc = DescTools: :CCC (comparison\$truth, comparison\$response, $\mathrm{ci}=$ "z-transform", conf.level $=0.95$, na.rm=TRUE) $\$ r h o . c$

CCC

\#\# est lwr.ci upr.ci

$\begin{array}{llllll}\text { \#\# } & 1 & 0.7181705 & 0.6954837 & 0.7394268\end{array}$

\#\#bias

bias (comparison\$response, comparison\$truth)

\#\#[1] -0.02806462

\#\#RMSE

rmse(comparison\$truth, comparison\$response) 
\#\#Transform log10 to normal values

comparison\$log10ksat1<- $10^{\wedge}$ comparison\$truth

comparison $\$ r e s p o n s e 1<-10^{\wedge}$ comparison $\$ r e s p o n s e$

\#\#Hexbin Plot

hexbinplot(log10ksat1 response1,

panel $=$ function $(x, y, \ldots)\{$

panel.hexbinplot $(x, y, \ldots)$

panel.abline $(c(0,1))$

data =comparison, $x l a b=$ "Predicted Ksat $[\mathrm{cm} /$ day $] "$,

$\mathrm{ylab}=$ "Measured Ksat $[\mathrm{cm} /$ day $] "$, cex.axis $=4$,

aspect="1", xbins=39.4, colramp = function $(n)$

\{viridis (8, alpha $=1$, begin $=0$, end $=1$,

direction $=-1$, option $=" \mathrm{C} ")\}$,

$x \lim =c(1,10000), \operatorname{ylim}=c(1,10000)$,

scales $=$ list (

$x=\operatorname{list}(\log =10$, equispaced.log $=$ FALSE),

$y=\operatorname{list}(\log =10$, equispaced.log $=$ FALSE)

),

font. $1 \mathrm{ab}=6$, cex.labels $=1.2$, font.axis $=2$, colorcut $=c(0,0.01,0.03,0.07,0.15,0.25,0.5,0.75,1))$ 


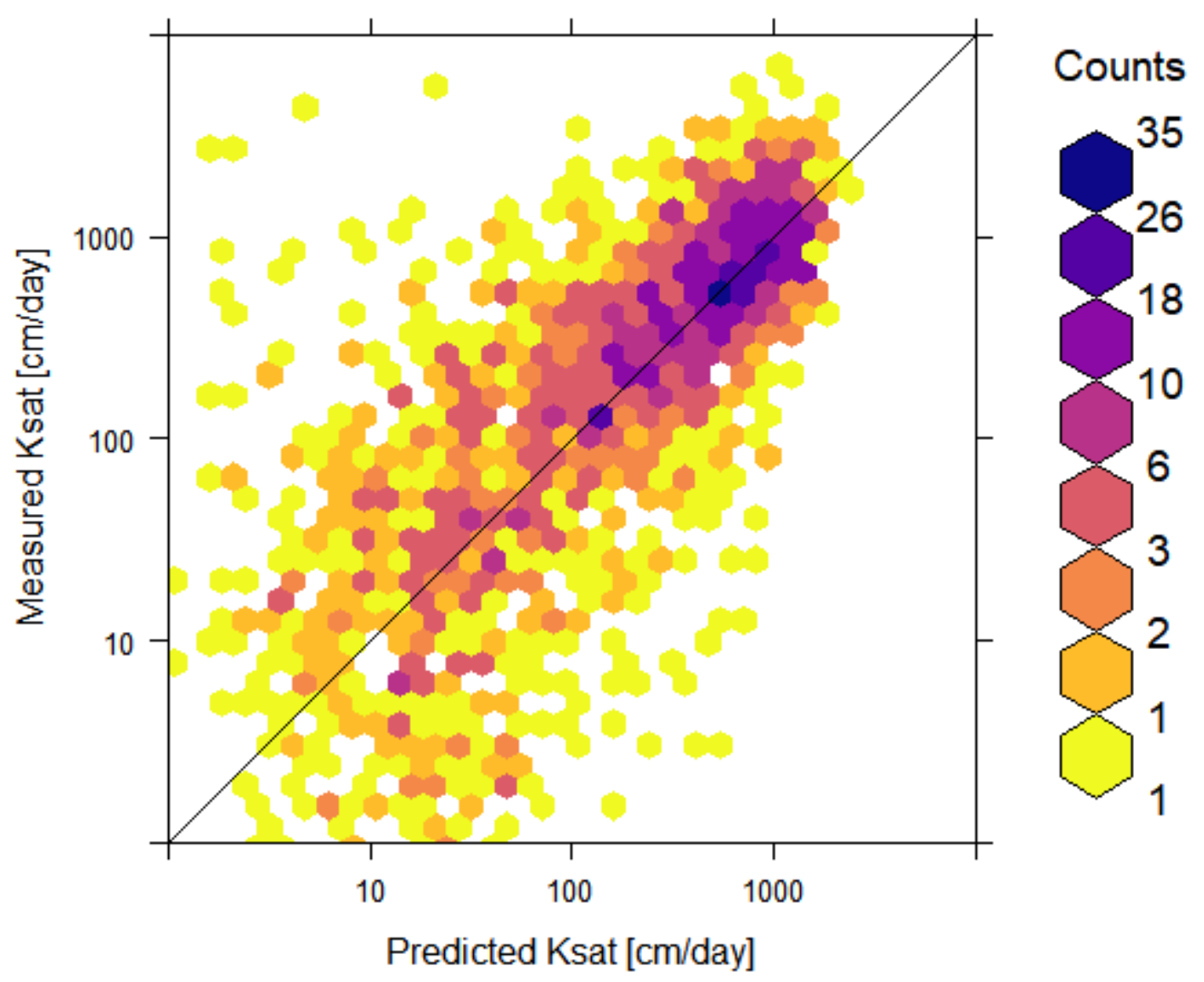

\#\#variable importance_selection

Var.ksat <- as.formula (paste("log_ksat clay_tot_psa + db_od + sand_tot_psa")) Var.ksat

\#\# log_ksat clay_tot_psa + db_od + sand_tot_psa 
Temperate.ksat <- PTF_lab[complete.cases(PTF_lab[,all.vars(Var.ksat)]), ]

$\mathrm{m} . \mathrm{ksat}<-$ randomForest(Var.ksat, Temperate.ksat, num.trees $=85$, $\mathrm{mtry}=3$, quantreg $=$ TRUE)

m.ksat

\#\#

\#\# Call:

\#\# randomForest $($ formula $=$ Var.ksat, data $=$ Temperate. $\mathrm{ksat}$, num.trees $=85, \quad$ mtry $=3$, quantreg $=$ TRUE $)$

\#\# Type of random forest: regression

Number of trees: 500

\#\# No. of variables tried at each split: 3

\#\#

\#\# $\quad$ Mean of squared residuals: 0.4335024

\#\# \% Var explained: 55.54

$\operatorname{varImpPlot}(m . k s a t$, sort=TRUE, $n \cdot \operatorname{var}=\min (3, \operatorname{nrow}(m . k s a t \$ i m p o r t a n c e)))$ 


\section{m.ksat}

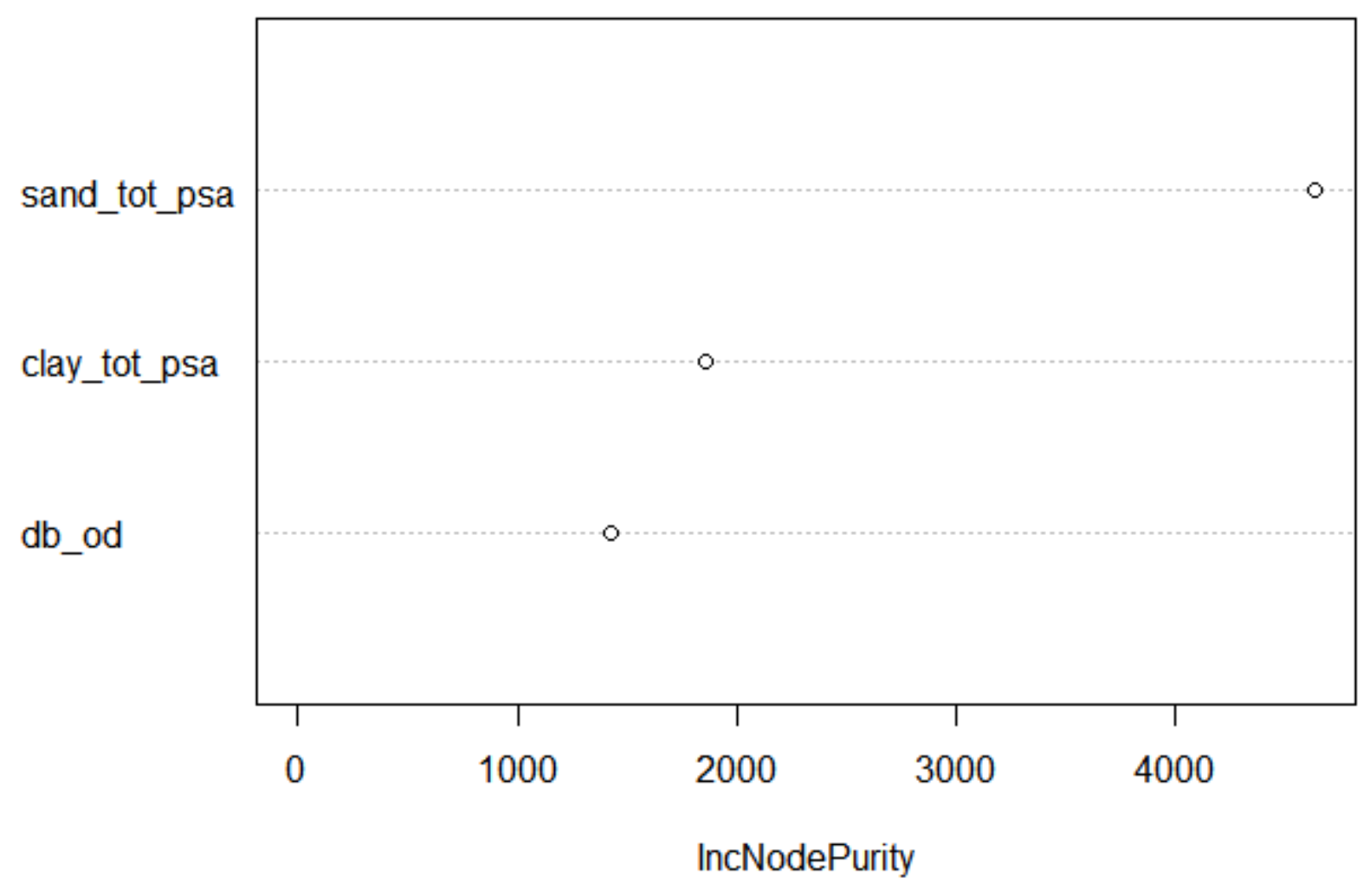

\section{Lab PTF tested on field dataset}

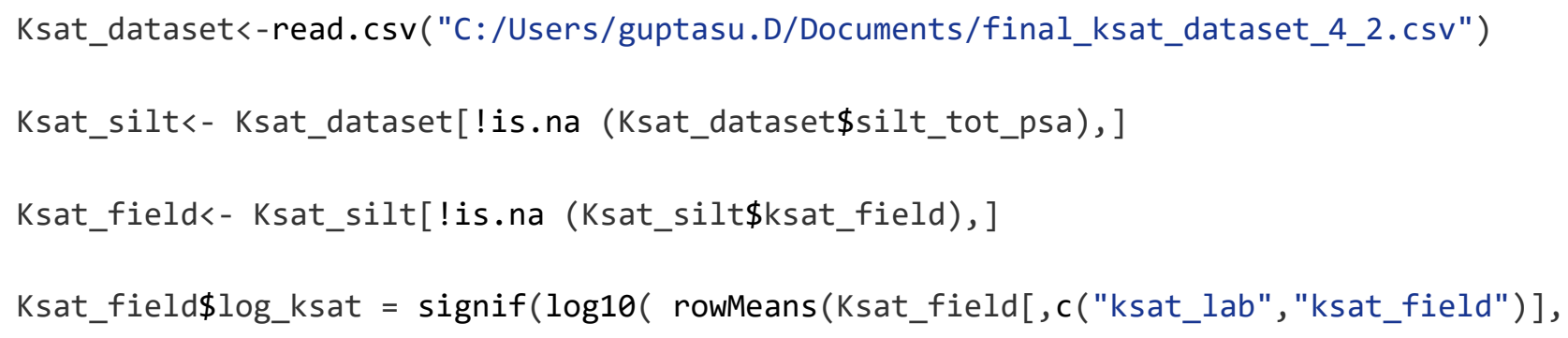




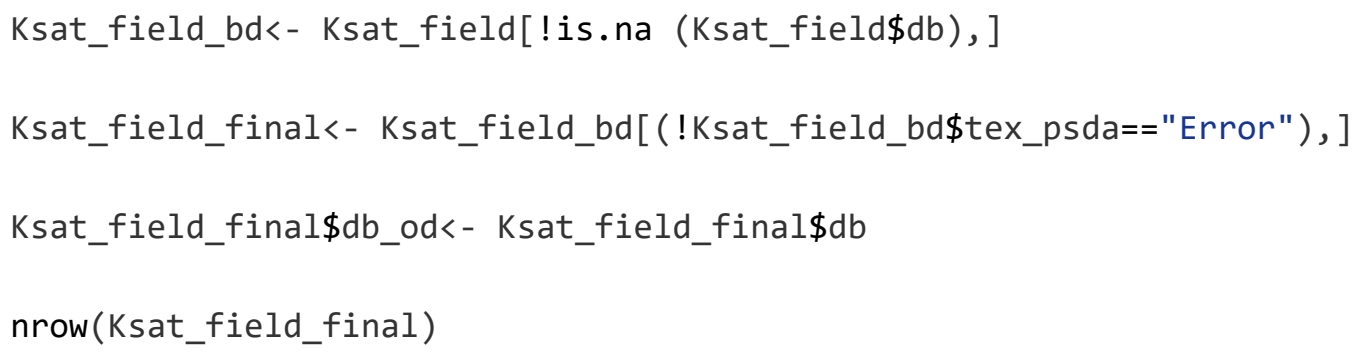


\#\#RMSE

rmse(y\$log_ksat, y\$response)

\#\# [1] 1.18587

\#\#Transform $\log 10$ to normal values

y\$log_ksat1<- 10^y $\$$ log_ksat

y\$response1<- $10^{\wedge} y \$$ response

hexbinplot(log_ksat1 response1,

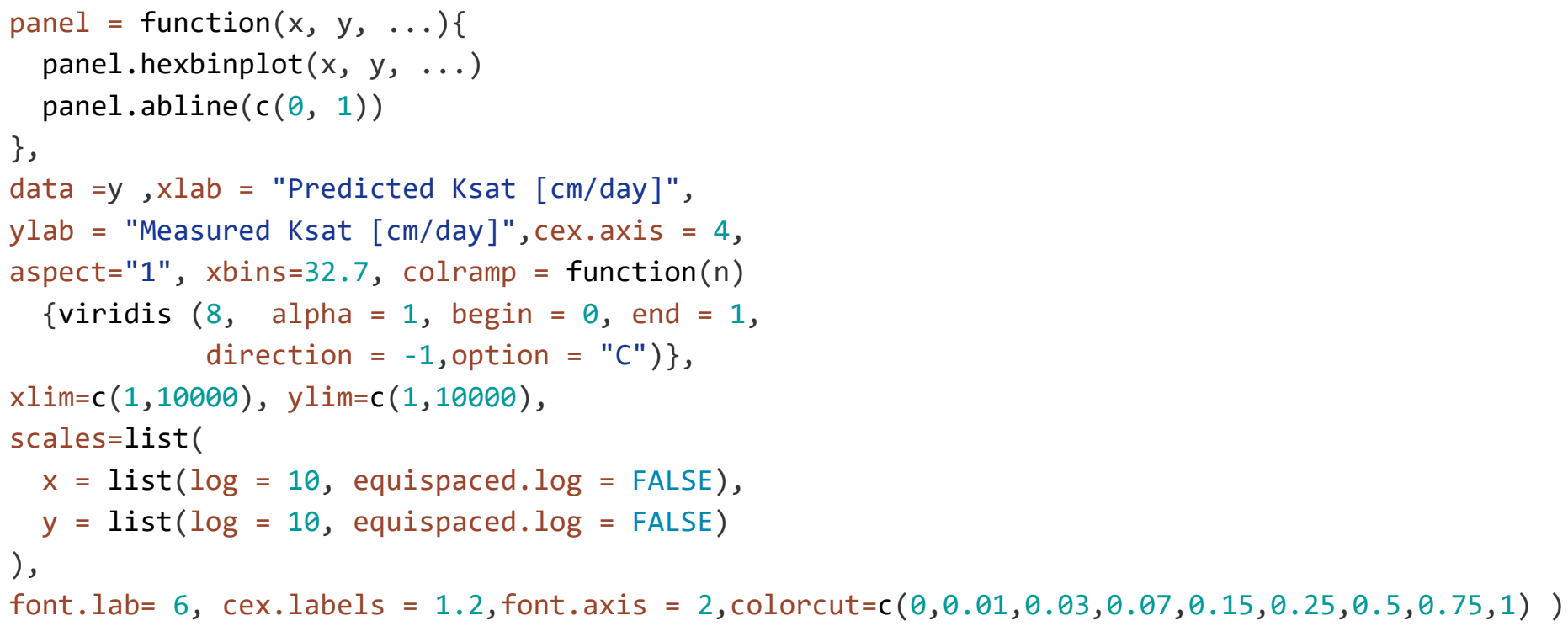




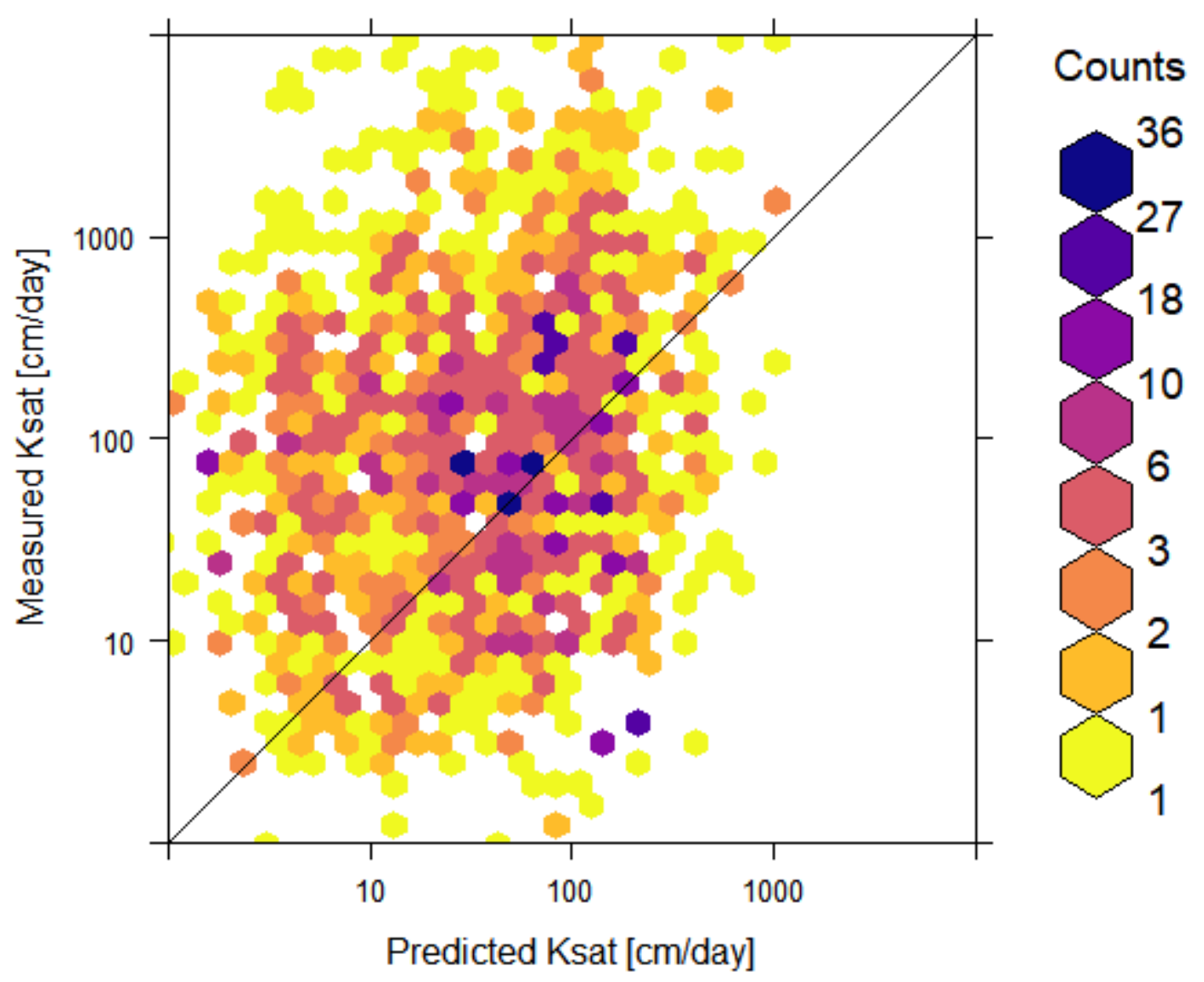

